\title{
How to Create Animal Models Using Genetic Knowledge?
}

\author{
Michel Bourin* \\ Neurobiology of anxiety and mood disorders, University of Nantes, France
}

Received: March 29, 2018; Accepted: May 25, 2018; Published: May 29, 2018

*Corresponding author: Michel Bourin, Neurobiology of anxiety and mood disorders, University of Nantes, France, Email: Michel.bourin@univnantes.fr

\begin{abstract}
New animal models have emerged in recent years; some are based on the possibility of genetic modifications as close as possible to those found in humans. We chose to show what could be achieved through genetic transcription. We chose 3 models: APP and APPxPS1 transgenic mice, frontotemporal dementia and tau mutations in the microtubule-associated protein tau and $\alpha$-synuclein accumulation in Lewy body diseases.
\end{abstract}

Key words: Genetic Models; Transgenic Mice; Tau Mutations; A -Synuclein;

\section{Introduction}

Animal models have been used to address a wide variety of scientific fields, from basic research to drug development and evaluation. The use of animals relies not only on the many biological similarities observed in most mammals, but also on the fact that human diseases often affect other animal species. This is particularly the case for most infectious diseases but also for very common diseases such as type I diabetes [1], hypertension, allergies, cancer, epilepsy, myopathies [2] and many others. Not only are these diseases shared, but the mechanisms are also so close that $90 \%$ of the veterinary drugs used to treat animals are identical or very similar to those used to treat humans. Many major advances in basic or medical research have been possible thanks to observations and tests on animals.In all cases, the animal model is essential to understand certain elements of a disease, to test innovative therapies, or even to reinvest traditional treatments to know the new possibilities and limits. Far from being the Alpha and Omega of biological research, the animal is nevertheless a central link, essential in the medical procedure which consists, for any new treatment, to evaluate the benefit/risk ratio for the patient.

\section{Natural models}

It has long been believed that there are natural patterns of human neurodegenerative diseases. Numerous neurological phenotypes, such as those observed in the mouse lines Reeler, Weaver and Staggerer, presenting disorders of balance and gait, have indeed been identified and then selected in farm animals. The lesions, related to a loss of function of the mutated protein, mainly concern the development of the cerebellum. Contrary to what one might have thought, these three lineages are not models of human ataxia. The mutation of the Reln gene [3] causes an abnormality of neuronal migration responsible for cerebellar lesions in the Reeler mice but, in humans, lissencephaly [4]. Reelin - the product of the Reln gene - has also been implicated in cases of schizophrenia [5]. In the Weaver mouse, cerebellar grain cells and dopaminergic neurons of substantia nigra are selectively affected $[6,7]$. The nonsense mutation concerns the gene Girk2, but the search for a mutation in the homologous human gene has hitherto proved to be negative. In Staggerer mice, atrophy of the cerebellum is due to loss of Purkinje cells and cells of the seed layer. The staggerer gene encodes the transcription factor ROR $\alpha$, a nuclear receptor [8] whose mutation has never been identified in humans. In conclusion, the phenotypes of the natural models evoked those of the human affections, and this similarity suggested that the selected lines could be useful for the research of physiopathological mechanisms [9]. These were mostly false leads, and most predictions proved to be erroneous, demonstrating, once again, that phenotypic analogy does not mean mechanistic kinship.

\section{Strategies for developing animal models}

The introduction of a mutated human gene or its inactivation in a laboratory animal is a more direct approach than the identification of natural phenotypes. The mouse is the animal of choice. It is easier to handle and maintain than the rat, let alone the primate, and the murine and human genomes are relatively close in structure. Additional transgenesis, the first method developed, is rarely ideal: blind insertion of multiple copies of the gene; sometimes massive overexpression of the protein that it codes; possible and unforeseen modification of embryonic development; sometimes abnormal expression topography, conditioned by the promoter associated with the transgene or by a natural promoter depending on the insertion site; variable effects according to the genetic background; persistence of wild gene expression. The alterations observed in the transgenic animal therefore often lead to choosing between two alternative interpretations: a real pathophysiological mechanism or translation of an artificial pathway of degradation in relation to an overproduction or an ectopic production of the protein 
in question? Homologous recombination [knock-in, KI], which invalidates the wild-type gene and places the transgene under the control of the natural promoter, or conditional expression to control the timing of transgene expression, alleviates some of the disadvantages mentioned above. Transgenic models, even evolved, do not reproduce all aspects of the human disease that usually involves a network of proteins interacting partners: the probability that all the protein partners of a species [the mouse] have the same reciprocal affinities that human molecules is indeed very weak. The chance to mimic an effect diminishes with the number of interactions that separates it from the mutated protein. Finally, the expression topography of the protein is often different in animals and humans, and depends on the promoter of the transgene.

\section{Transgenic models of diseases characterized by protein accumulation}

Several degenerative disorders are characterized by the accumulation, intra- or extracellularly, of an abnormally aggregated protein. We will take three examples:

\section{APP and APPxPS1 transgenic mice: study amyloidogenesis observed in Alzheimer's disease}

The brain lesions of Alzheimer's disease consist of senile plaques and neurofibrillary tangles. The senile plaques include an amyloid heart, which accumulates the $A \beta$ peptide derived from the cleavage of a precursor, the amyloid protein precursor [APP]. Neurofibrillary tangles are mainly composed of tau protein [see below]. The mutations responsible for the hereditary Alzheimer's diseases identified today affect the gene of APP, or that of proteins involved in its metabolism: presenilin 1 or presenilin 2. The hypothesis of the "amyloid cascade" assumes that the alterations of the tau protein are secondary. Experimentally, amyloid deposits are produced in the cerebral parenchyma and the vessel wall by a human APP transgene carrying one or more mutations. The association of the mutated gene with preseniline accelerates the onset and severity of deposits. APP mice crossed with animals carrying a mutated recombination PS1 gene [PS1 $\mathrm{KI}]$, produce even higher amounts of $A \beta$ peptide, associated with a selective neuronal loss in Ammon's horn [10]. In APPxPS1 mice, extracellular deposits and intracellular accumulations of $A \beta$ peptide in the form of granules are observed as early as 3 months of age [11]. The formation of amyloid deposits could be followed in vivo using a multiphoton microscope through a window in the cranial box. It is still too early to determine whether intracellular granules containing the $A \beta$ peptide testify to a pathogenic mechanism applicable to humans, or should be considered as a consequence of the massive and artificial overexpression of APP. Tau pathology could never be reproduced satisfactorily in APP or APPXPS1 animals. The link between the extracellular accumulation of $A \beta$ peptide and that, intracellular, tau protein remains poorly understood, and has led to the production of mice expressing both a tau transgene and APP. In these lines, senile plaques have been observed with, as in humans, an amyloid center and positive extensions for tau. The intracerebral injection of $A \beta$ peptide in tau mice favored the appearance of neurofibrillary tangles in the cell body of neurons whose axons were projected on the injection site [12]. APP mice are used today to test the activity of new treatments aimed at reducing $A \beta$ peptide deposits, such as vaccination by injection of exogenous $A \beta$ peptide. On the other hand, they do not make it possible to determine the impact of the new therapeutics on the tau pathology, badly reproduced in the simple transgenic models or artificially recreated in the doubly transgenic lines also comprising a mutation of the tau protein.

\section{Frontotemporal dementia and tau mutations in the microtubule-associated protein tau}

Mutations in the microtubule-associated protein tau [MAPT] gene, encoding tau, are associated with frontotemporal dementia with Parkinson's syndrome.These inherited "taupathies" are characterized by polymorphic intracellular accumulations of tau protein in neurons [neurofibrillary tangles] and glia $[13,14]$. Tau proteins bind to microtubules that stabilize and promote polymerization. The binding domain comprises, according to the splicing, 3 or 4 repetitive segments. Phosphorylation of tau prevents its interaction with microtubules. About 20 mouse lines were produced with a tau transgene comprising 3 or 4 repetitive segments and various mutations. The promoters used have often induced a particularly marked overexpression of tau protein in the motor neuron, which explains why the clinical phenotype is driving, probably related to an alteration of axoplasmic transport [15]. Tau protein accumulates in hyperphosphorylated form mainly in the somatodendritic compartment of the neuron [16]. The normal, axonal subcellular topography of tau is conditioned by the targeting of its mRNA at the base of the axon, due to a localized sequence in its noncoding part. The presence of neurofibrillary tangles has been reported in a transgenic line obtained by homologous recombination of the normal human tau protein [17]. A conditional tau transgene has shown that the suppression of transgene expression does not hinder the progression of neurofibrillary tangles, suggesting that the tau pathology may, once established, spontaneously maintain itself. The complexity of the MAPT gene, its splicing and its subcellular addressing explains the difficulties encountered in producing neurofibrillary tangles or glial inclusions similar to those observed in humans.

\section{$\alpha$-synuclein accumulation in Lewy body diseases}

Lewy which body diseases have a wide range of clinical expressions includes motor syndrome [prominent in Parkinson's disease] and cognitive impairment [prominent in Lewy body dementia][18]. Lewy bodies are spherical eosinophilic inclusions that sit in the cell body of the neuron, and are fibrillar in electron microscopy. Various missense mutations have been identified in the gene encoding $\alpha$-synuclein, a synaptic protein, in patients with the familial form of Parkinson's disease [19]. They led to the discovery of $\alpha$-synuclein in Lewy bodies, the latter also containing ubiquitin, which binds to proteins to be destroyed by the proteasome. In transgenic mice for the mutated form of $\alpha$-synuclein the overexpressed protein fills the somatodendritic or axonal compartment of the cell. Aggregations of $\alpha$ synuclein have also been demonstrated [20]. Only certain lines develop 
a phenotype characterized by paralysis of extremities or sensorimotor abnormalities, different from those of human Parkinson's disease and in relation to the cortical and subcortical topography of transgene expression. . The crossing of these $\alpha$-synuclein transgenic mice with APP mice potentiates the accumulation of $\alpha$-synuclein, which can then assume a fibrillar aspect. This interaction between Alzheimer's and Parkinson's pathologies, observed in humans, remains poorly understood.

\section{Conclusion}

The creation of animal models using genetics is promising for creating drugs or treatments in diseases that are difficult to observe in animals. It is the mental or neurological diseases that have the most to gain from this perspective.

In just a few years, the progress made by molecular genetics has revolutionized the knowledge of neurodegenerative diseases: the neurologist was looking for a symptom [dementia, aphasia, cerebellar syndrome] that would one day enable him to better understand the mechanism of these affections. It has to be said that none of the recent discoveries come from the study of symptoms [or, what amounts to the same thing, from the topography of lesions]. The role of Huntingtin, $A \beta$ peptide, $\alpha$-synuclein or tau protein has been suspected by genetic analysis of families and isolation of accumulated proteins; none of them was identified by screening the proteins expressed specifically in the most affected structures, those whose lesions caused chorea, dementia or Parkinson's syndrome. Moreover, in animal lines spontaneously presenting a symptom similar to that observed in a human disease, the mutations were not those identified in humans.

\section{Refernces}

1. Nobelprize.Org - The discovery of insulin. is available at www nobelprize.org

2. Klug MG, Soonpaa MH, Koh GY, Field LJ. Genetically selected cardiomyocytes from differentiating embronic stem cells form stable intracardiac grafts. J Clin Invest. 1996;98(1):216-224.

3. Castagna C, Merighi A, Lossi L. Cell death and neurodegeneration in the postnatal development of cerebellar vermis in normal and Reeler mice. Ann Anat. 2016;207:76-90. doi: 10.1016/j.aanat.2016.01.010

4. Fry AE, Cushion TD, Pilz DT. The genetics of lissencephaly. Am J Med Genet C Semin Med Genet. 2014;166C(2):198-210. doi: 10.1002/ ajmg.c.31402

5. Kohno T. Regulatory Mechanisms and Physiological Significance of Reelin Function. Yakugaku Zasshi. 2017;137(10):1233-1240. doi: 10.1248/yakushi.17-00127

6. Cendelin J. From mice to men: lessons from mutant ataxic mice. Cerebellum Ataxias. 2014;1:4. doi: 10.1186/2053-8871-1-4
7. Maricich SM, Soha J, Trenkner E, Herrup K. Failed cell migration and death of purkinje cells and deep nuclear neurons in the weaver cerebellum. J Neurosci. 1997;17(10):3675-3683.

8. Patil N, Cox DR, Bhat D, Faham M, Myers RM, Peterson AS. A potassium channel mutation in weaver mice implicates membrane excitability in granule cell differentiation. Nat Genet. 1995;11(2):126-129.

9. Gold DA, Baek SH, Schork NJ, Rose DW, Larsen DD, Sachs BD, et al. ROR $\alpha$ coordinates reciprocal signaling in cerebellar development through sonic hedgehog and calcium-dependent pathways. Neuron. 2003;40(6):1119-1131.

10. Blanchard V, Moussaoui S, Czech C, Touchet N, Bonici B, Planche $\mathrm{M}$, et al. Time sequence of maturation of dystrophic neurites associated with A $\beta$ deposits in APP/PS1 transgenic mice. Exp Neurol. 2003;184(1):247-263.

11. Schmitz C, Rutten BP, Pielen A, et al. Hippocampal neuron loss exceeds amyloid plaque load in a transgenic mouse model of Alzheimer's disease. Am J Pathol. 2004;164(4):1495-502.

12. Langui D, Girardot N, El Hachimi H, Bernadette Allinquant, Véronique Blanchard, et al. Subcellular topography of neuronal A $\beta$ peptide in APPxPS1 transgenic mice. Am J Pathol. 2004;165(5):1465-1477.

13. Götz J, Chen F, van Dorpe J, Nitsch RM. Formation of neurofibrillary tangles in P301L tau transgenic mice induced by A 342 fibrils. Science. 2001;293 (5534):1491-1495.

14. Lebouvier T, Pasquier F, Buée L. Update on tauopathies. Curr Opin Neurol. 2017;30(6):589-598. doi: 10.1097/WC0.0000000000000502

15. Ghetti B, Hutton ML, Wszolek ZK. Fronto-temporal dementia and parkinsonism linked to chromosome 17 associated with Tau gene mutations [FTDP-17T]. In: Dickson D, ed. Neurodegeneration: the molecular pathology of dementia and movement disorders. Basel: ISN Neuropath Press. 2003:86-102.

16. Probst A, Gotz J, Wiederhold KH, et al. Axonopathy and amyotrophy in mice transgenic for human four-repeat tau protein. Acta Neuropathol [Berl]. 2000;99(5):469-481.

17. Andorfer C, Acker CM, Kress Y, Hof PR, Duff K, Davies P. Cell-cycle reentry and cell death in transgenic mice expressing nonmutant human tau isoforms. J Neurosci. 2005;25(22):5446-5454.

18. Wong YC, Krainc D. $\alpha$-synuclein toxicity in neurodegeneration: mechanism and therapeutic strategies. Nat Med. 2017;23(2):1-13. doi: 10.1038/nm.4269

19. Saito Y. DJ-1 as a Biomarker of Parkinson's disease. Adv Exp Med Biol. 2017;1037:149-171. doi: 10.1007/978-981-10-6583-5_10

20. Nakamura K, Mori F, Kon T, Tanji K, Miki Y, Tomiyama M, et al. Filamentous aggregations of phosphorylated $\alpha$-synuclein in Schwann cells [Schwann cell cytoplasmic inclusions] in multiple system atrophy. Acta Neuropathol Commun. 2015;21;3:29. doi: 10.1186/s40478-0150208-0 